\title{
Reading the DNA Methylation Signal
}

\author{
A. BiRd AND D. MACleod \\ Wellcome Trust Centre for Cell Biology, University of Edinburgh, \\ Edinburgh EH9 3JR, Scotland, United Kingdom
}

A cell's properties depend largely on its pattern of gene expression. During the development of complex multicellular organisms, multiple cell lineages emerge among the descendants of the single zygotic cell. Within each lineage, the range of gene expression programs is effectively restricted, culminating in the definitive program of the final differentiated cell. As part of this process, some genes are selected for future activity, whereas others are disqualified by long-term silencing. For a specific gene in one cell type, silence versus activity is not determined solely by the available combinations of transcripion factors in that cell, but also by local differentiation of chromatin structure near to or including the gene. A key feature of such local differentiation of the genome is its stability within and between cell generations. The study of these processes has come to be known as epigenetics. Contemporary epigenetics might therefore be defined as, "the study of the structural adaptation of chromosome regions so as to perpetuate local activity states."

Three sorts of epigenetic system have traditionally been studied: DNA methylation, the polycomb/trithorax system, and histone modification or variant substitution. Other epigenetic categories have emerged, however, and are the subject of intense study as reflected elsewhere in this volume. They include silencing based on noncoding RNAs, trancriptional silencing triggered by doublestranded RNA interference, and silencing or activity that responds to localization of genes within the nucleus. All of these processes appear to be closely interwoven with histone modification, which is itself a diverse and complex system of chromosome marking. For each system there is evidence that the trigger for altered chromatin is a preexisting transcriptional state. In other words, it appears to be the transcripion factors that primarily determine whether a gene is active or inactive, after which chromatin structure is adapted accordingly and the state is memorized. This "secondary" status of many epigenetic mechanisms does not diminish their importance. Stable, leakproof gene silencing requires sophisticated mechanisms for sensing the transcriptional status quo without perturbing it and then passing the memory between cell generations without disruption by DNA replication.

Obviously the most direct way of marking genes either for silence or activity would be to apply chemical tags to the DNA itself. In practice, however, the only widespread modification of DNA is methylation, either of the C5 position of the cytosine ring or of the N6 position of the adenine ring. Other modifications are found, but have limited phylogenetic distribution. In multicellular eukaryotes, only $\mathrm{m}^{5} \mathrm{C}$ is convincingly reported. A plausible explanation for the narrow spectrum of DNA modifications is that chemical moieties, such as acetyl or phosphate groups, or methylation at other positions within the DNA structure would interfere with aspects of DNA function. Therefore the scope for chemically tagging genomic DNA, without compromising its essential information coding and storage properties, may be extremely limited. Even $\mathrm{m}^{5} \mathrm{C}$, which does not alter the base-pairing specificity of cytosine and has survived several billion years of scrutiny by natural selection, has drawbacks, as it is a mutagenic base in vertebrates and bacteria (Coulondre et al. 1978; Bird 1980). This trade-off between benefit and disadvantage may explain the evolutionary volatility of cytosine methylation across evolutionary time. Some organisms have dispensed with DNA methylation whereas their relatives retain it. For example, the fungus Neurospora crassa displays cytosine methylation, but the fission and budding yeasts-also fungi- do not.

The emergence of complex gene expression programs in multicellular organisms with functionally adapted cell types may have depended on the ability to epigenetically mark genes for either activity or silence during development. Without an effective memory system to enforce the activity state established at critical developmental stages, it would be necessary to constantly reiterate the initial conditions in order to reestablish the program. DNA is the obvious substrate for memorable marks of this kind, but, as pointed out above, has a limited capacity for chemical modification. This shortcoming of the genetic material may have provided a driving force behind the evolution of histones. Rather than marking the DNA itself, why not coat it uniformly with proteins that are susceptible to a plethora of chemical modifications (Jenuwein and Allis 2001)? A precondition of such a system is that histones do not move around. If they did, marks would not be locus specific and would lose their value. Histone loss by degradation or stochastic processes would also need to be compensated by insuring that replacement histones acquired modifications that are appropriate to the region. What we know about histones supports these requirements. The half-life of histones on DNA appears to be long and there is evidence for processes that transfer modification from one nucleosome to its (perhaps new) neighbor. An example is the interaction of HP1 with both methylated lysine 9 of histone $\mathrm{H} 3$ (H3K9) and the histone methyltransferase Suvar 3.9 (Bannister et al. 2001). Re- 
cruitment of $\mathrm{H} 3 \mathrm{~K} 9$ methyltransferase to an already methylated histone by HP1 may facilitate the spreading of this modification to neighboring H3 histones.

\section{READING EPIGENETIC MARKS}

For any epigenetic system, there are two key questions to be answered. (1) What creates patterns of chromatin marking? (2) How are the marks read by the cell to generate biological outcomes? Although this article focuses on DNA methylation and addresses the second question (how is the DNA methylation signal read?), similar questions apply to the decoding of histone marks. Two kinds of model have been advanced for the functional interpretation of histone modifications (methylation, phosphorylation, acetylation etc.): Either (1) the marks affect the properties of nucleosomal chromatin directly, for example, by changing histone DNA contacts; or (2) histone modifications attract proteins that recognize and bind to the modified (or unmodified) site and mediate downstream biological effects. Proteins that bind modified histones are comparable to methyl-CpG binding proteins, as both interpret chemical marks on chromatin. They include bromodomain proteins, which recognize acetylated lysine in histone tails (Winston and Allis 1999). Whereas bromodomain proteins are associated with gene activity, chromodomain proteins promote silencing and are found to interact with methylated lysine residues on K9 of histone H3 (Bannister et al. 2001; Lachner et al. 2001). At present, it is not known how many histone modifications have dedicated binding proteins to interpret their biological message.

As may be the case for repressive histone marks, methylation of cytosine in the dinucleotide $\mathrm{CpG}$ is known to repress transcription in two different ways: by repelling transcription factors or by attracting proteins that bring about transcriptional repression. There are examples of factors that cannot bind productively to DNA when there is a methyl-CpG within their binding site. An example is the protein CTCF that binds to chromatin boundaries. Its importance in the regulation of the Igf2H19 imprinted domain is now well documented (Bell and Felsenfeld 2000; Hark et al. 2000). Likewise, we know of several proteins that can bind to methyl-CpG and bring about transcriptional repression in model reporter gene systems (Bird and Wolffe 1999), but have only begun to learn about endogenous genes that appear to be regulated by these proteins in normal cells.

DNA methylation has two well-documented biological consequences. On the negative side, it causes mutations due to inefficient repair of G:T mismatches that arise when $\mathrm{m}^{5} \mathrm{C}$ becomes spontaneously deaminated. More positively, it causes local, heritable transcriptional silencing. Of the five known methyl-CpG-binding proteinsMbd1, Mbd2, Mbd4, MeCP2, and Kaiso (Fig. 1)-Mbd4 has been clearly shown to mediate repair of $\mathrm{m}^{5} \mathrm{C}$ mutations (Hendrich et al. 1999; Millar et al. 2002), whereas the other four proteins are implicated in transcriptional silencing (Bird and Wolffe 1999). This review will discuss current knowledge about these transcriptional repressors, based on work of this laboratory and others.

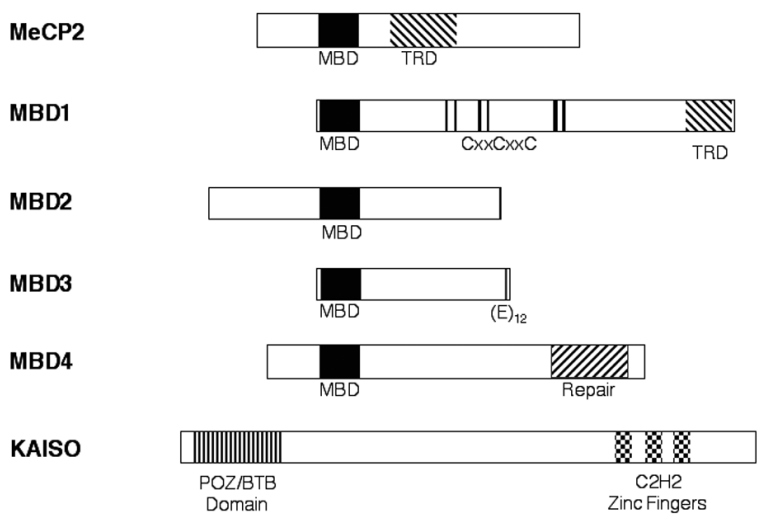

Figure 1. The methyl-CpG-binding proteins. Five of these proteins are related by possession of a methyl-CpG-binding domain (MBD), although in the case of MBD3 this domain does not show a strong preference for binding to methyl-CpG sites. Kaiso is an unrelated protein that binds to $\mathrm{m}^{5} \mathrm{CGm}^{5} \mathrm{CG}$ sites via its zinc finger domains. TRD indicates the transcriptional repression domains of MBD1 and MeCP2.

\section{REPRESSION MEDIATED BY MBD PROTEINS}

Evidence for "indirect" transcriptional repression by proteins that recognize methylated DNA was first obtained using in vitro transcription in nuclear extracts (Boyes and Bird 1991). In contrast to transfection of DNA into cultured cells, which invariably leads to silencing, methylated DNA could be transcribed as efficiently as nonmethylated DNA in extracts from these same cells. Specific repression did become apparent, however, when the amount of methylated DNA template was reduced below a threshold level. This repression was relieved by adding excess methylated DNA competitor, reinforcing the view that something in the extracts was able to bind to and silence methylated DNA. Prior to this study, an activity that bound multiple methylated sites was detected in nuclear extracts by bandshift assays (see Fig. 2) (Meehan et al. 1989). The properties of the putative repressor matched those of the "MeCP" (for MethylCpG-binding Protein) activity. In particular, F9 teratocarcinoma cells, which lacked the methyl-CpG binding activity, also showed unprecedentedly weak repression of methylated reporter genes (Boyes and Bird 1991; Levine et al. 1992). It was concluded that DNA methylation-mediated transcriptional repression depends heavily on methyl-CpG-binding activity.

Attempts to purify MeCP led to the isolation of a protein that was clearly different from the activity originally detected by bandshift assays (Lewis et al. 1992). The new protein was therefore christened MeCP2, to distinguish it from the initial activity, which by default became known as MeCP1. Deletion analysis showed that MeCP2 bound methylated sites via a methyl-CpG binding domain (MBD) of about 80 amino acids (Nan et al. 1993). The three-dimensional structure of the free MBD was determined by NMR (Ohki et al. 1999; Wakefield et al. 1999), soon to be followed by a structure of the MBDDNA complex (Ohki et al. 2001). Database searching picked out proteins that contained domains related to the 


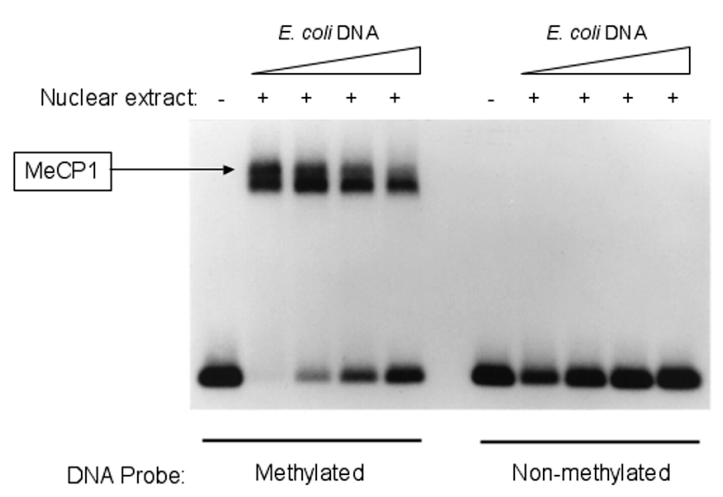

Figure 2. Detection of a methyl-CpG-binding activity in nuclear extracts from mouse liver. The upper DNA protein complex bands include the MeCP1 complex, whose DNA-binding component is Mbd2. The CG11 probe DNA is methylated at multiple CpG sites (left lanes) or is unmethylated (right lanes).

MBD of MeCP2 and several of these (Mbd1, Mbd2, and Mbd4) were shown to bind methylated DNA (Cross et al. 1997; Hendrich and Bird 1998). Methylated DNA binding in vivo was demonstrated by assaying localization of exogenous MBD proteins to heterochromatic foci of mouse nuclei. These foci contain the mouse major satellite, which happens to possess $\sim 40 \%$ of all methyl-CpG in the mouse nucleus. Mutations in the DNA methyltransferase gene Dnmt1 that greatly reduce genomic CpG methylation ( $\mathrm{Li}$ et al. 1992) prevent efficient localization of MeCP2 (Nan et al. 1996), Mbd1, Mbd2, and Mbd4 (Hendrich and Bird 1998), confirming the need for methyl-CpG as their in vivo chromosomal target.

What about the function of these proteins in the cell? Attempts to answer this question are still ongoing, but much is already known. As the first MBD protein to be purified, $\mathrm{MeCP} 2$ led the way in functional studies. DNA methylation-dependent transcriptional repression was established using in vitro extracts and transient transfections (Nan et al. 1997). In this way, a transcriptional repression domain was identified. A significant advance in understanding occurred when our group and the group of Alan Wolffe showed that the TRD of MeCP2 recruited the Sin3A-histone deacetylase corepressor complex (Jones et al. 1998; Nan et al. 1998). Repression by $\mathrm{MeCP} 2$ could be relieved by the histone deacetylase inhibitor trichostatin A. These studies provided a molecular explanation for the long suspected link between DNA methylation and a repressive chromatin structure. Since then there has been progress in characterizing all MBD protein complexes. Interestingly, each MBD protein appears to specialize by associating with a different corepressor complex. At the time of writing, the MBD1 complex is not fully described, but the involvement of MCAF suggests that a histone methyltransferase may be involved (Fujita et al. 2003). Unexpectedly, an isoform of Mbd1 turns out to have a second DNA-binding domain specific for nonmethylated $\mathrm{CpG}$, suggesting that this protein can potentially interpret $\mathrm{CpG}$ as a repressive signal whether it is methylated or not (Jorgensen et al. 2004). The biological rationale for this dual DNA-binding speci-

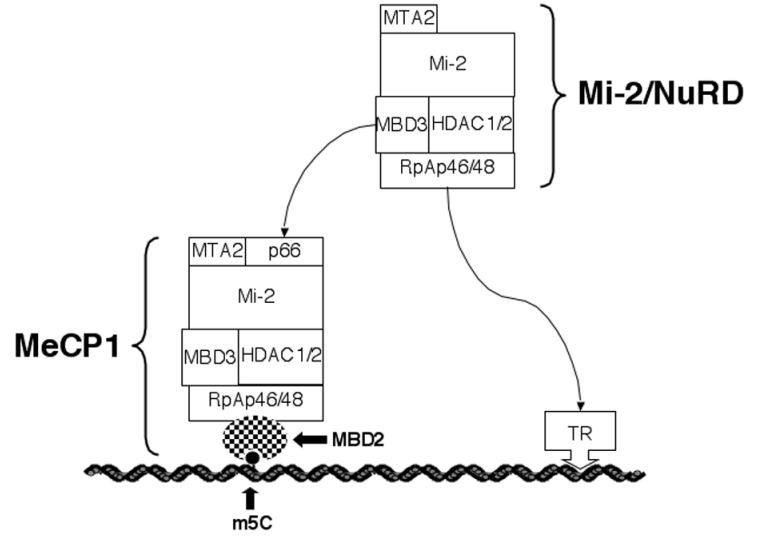

Figure 3. A diagramatic representation of the MeCP1 complex. The complex includes Mbd2 plus the NuRD complex. NuRD includes histone deacetylases and the chromatin remodeling protein $\mathrm{Mi}-2$. NuRD is recruited to DNA by a number of alternative DNA binding proteins (TR), of which Mbd2 is just one.

ficity is currently unknown. MBD2 associates with the NuRD or Mi-2 complex (Fig. 3) which contains histone deacetylases and the ATP-dependent chromatin remodeling protein, Mi-2 (Ng et al. 1999; Wade et al. 1999; Feng and Zhang 2001). The latter complex corresponds to MeCP1 (Fig. 2), the methyl-CpG-binding activity originally detected in nuclear extracts (Meehan et al. 1989).

A recent addition to the list of methyl-CpG-binding proteins is Kaiso, which is not a member of the MBD family (Prokhortchouk et al. 2001). This protein was identified biochemically as the basis of a methyl-CpGbinding activity in nuclear extracts and found to be identical to the Kaiso protein that was initially identified as a binding partner of p120 - itself a partner of the cell surface protein p120 (Daniel and Reynolds 1999). Kaiso is a POS-BTB domain protein in which the zinc fingers bind the sequence $5^{\prime} \mathrm{m}^{5} \mathrm{CGm}^{5} \mathrm{CG}$ with high affinity. It has also been reported that the isolated zinc finger domain of Kaiso is capable of binding to nonmethylated DNA sequences (Daniel et al. 2002). The involvement of Kaiso in repression of methylated genes in vivo was established by studies that identified the N-CoR corepressor complex as its binding partner (Yoon et al. 2003). The tantalizing link between Kaiso and cytoplasmic signaling via p120, as suggested by yeast two-hybrid and coimmunoprecipitation data, remains to be elucidated.

\section{TARGET GENES OF METHYL-CpG-BINDING PROTEINS}

Early studies of the function of methyl-CpG-binding proteins relied on model reporter gene systems. Next, it became important to assess their significance in cells of the organism by identifying target genes whose regulation is disrupted in the absence of a particular protein. Mouse gene knockouts are the system of choice for these studies. The first MBD protein gene to be disrupted was the X-linked Mecp2 gene (Chen et al. 2001; Guy et al. 2001). Two laboratories found that Mecp2-null mice are 
born and develop normally for several weeks, but they acquire a variety of neurological symptoms at about 6 weeks of age leading to death at $\sim 10$ weeks. This delayedonset phenotype, which is fully penetrant, recalls human Rett Syndrome, which is caused by $M E C P 2$ mutations (Amir et al. 1999; see below). Interestingly, deletion of the Mecp 2 gene only in mouse brain cells using cre expression driven by the brain-specific nestin promoter caused the same symptoms as deletion in the whole mouse (Chen et al. 2001; Guy et al. 2001). Therefore, although MeCP2 is ubiquitously expressed in cells of the mouse, the Mecp2-null phenotype appears to be entirely due to its absence in the brain. Biochemical and immunocytochemical studies established that MeCP2 expression levels are highest in the brain; specifically in neurons (Shahbazian et al. 2002).

Knockout of the $M b d 2$ gene in mice is compatible with viability and fertility, but some phenotypic effects were noticed (Hendrich et al. 2001). Mbd2-/- mothers failed to fully nurture their young, which consequently were underweight regardless of genotype. This weak maternal response to pups was also evident from the delayed retrieval of pups after they were removed from the nests of Mbd2-/- mothers. Other phenotypes were initially less obvious, but led to identification of the first target genes for a methyl-CpG binding protein. First, cells derived from $M b d 2-1-$ mice were found to be unable to repress methylated reporter genes effectively. This finding matched early experiments using in vitro extracts, which showed that the MeCP1 complex-whose DNA-binding component is $\mathrm{Mbd} 2$ - is largely responsible for the repression of methylated reporter genes.

This evidence that Mbd2-deficiency might compromise repression of methylated genes raised the possibility that a careful search might uncover misregulation of endogenous genes in these mice. Success came with an analysis of $\mathrm{T}$ cell differentiation in the mouse (Hutchins et al. 2002). Sorting of $M b d 2-/-$ cells by fluorescence allowed detection of a small fraction of naive and differentiated $\mathrm{T}$ cells in which expression of both interleukin 4 (Il4) and interferon gamma (If $\gamma$ ) genes was derepressed. Expression of Il4 and If $\gamma$ is normally mutually exclusive and is initiated by triggering differentiation into either the Th2 or the Th1 pathway, respectively. In the absence of Mbd2, differentiated Mbd2-/- thymocytes often expressed both genes and this misexpression effect could be enhanced by addition of the histone deacetylase inhibitor trichostatin A. Chromatin immunoprecipitation showed that Mbd2 was normally present at the Il4 promoter and was lost when the gene was transcriptionally activated. It is noteworthy that absence of $\mathrm{Mbd} 2$ does not lead to $100 \%$ reactivation of the inappropriate gene; the effect is instead stochastic in that only a fraction of all Th1 cells derepress If $\gamma$, but derepression in those cells appears to be complete. Thus the removal of Mbd2 appears to increase the normally very low probability that these silent genes will become fully reactivated. Similarly, reactivation of the aberrantly silenced pi-class glutathione S-transferase gene can be induced in cancer cell lines by Mbd2 deficiency (Lin and Nelson 2003). It seems likely that careful investigation of specific cell types will uncover fur- ther examples of the loosening of gene repression caused by absence of $\mathrm{Mbd} 2$.

The status of the Mecp2-null mouse as a model for Rett Syndrome has stimulated the search for MeCP2 target genes. Initial attempts to detect misregulated genes in the brains of the mutant animals offered partial success (Tudor et al. 2002). Deregulation of gene expression was apparent, but the degree of misexpression was subtle and only acquired statistical significance when groups of affected genes were considered together. The "candidate gene" approach proved more successful. Two groups (Chen et al. 2003; Martinowich et al. 2003) hypothesized that MeCP2 might play a role in activity-dependent expression of the gene for brain-derived neurotropic factor $(B d n f)$. Only one of several $B d n f$ promoters responds to calcium-dependent activation and this promoter was shown to associate with MeCP2 in cultured neurons. Upon stimulation of the neurons, MeCP2 became phosphorylated and was displaced from this promoter (Chen et al. 2003). Although the effect of MeCP2 deficiency on expression proved to be small in this in vitro system, it is possible that deregulation has important consequences in the animal itself. That MeCP2 deficiency can cause major gene expression changes was shown in a nonmammalian system, the embryo of the amphibian, Xenopus laevis. Here, MeCP2 was found associated with the promoter of the Hairy $2 a$ gene, whose product is important for limiting the number of embryonic cells that become neurons (Stancheva et al. 2003). Activation of the gene during development was accompanied by displacement of MeCP2, suggesting that, as in the case of the mouse $B d n f$ gene, MeCP2 is involved in the dynamic control of gene expression. These findings suggest that the view of methyl-CpG-binding proteins as long-term silencers of gene expression may need to be modified. It remains possible, however, that they may be involved in both stable and dynamic repression, depending on the locus concerned.

A target for Kaiso in cancer cell lines has clearly been shown to depend on recruitment of this protein plus the NCoR corepressor (Yoon et al. 2003), but MBD1 target genes are, at the time of writing, yet to be reported. Immunoprecipitation experiments have identified multiple DNA sequences associated with various MBD proteins (Ballestar et al. 2003), but the regulatory consequences of MBD protein withdrawal at these loci have not yet been assessed. It is possible that all genomic regions that associate with one of these proteins are to some extent regulated by them. Alternatively, MBD proteins may spend time unproductively at methyl-CpG sites where their regulatory input is superfluous. In depth study of specific genes is required to distinguish these possibilities. A related issue concerns the redundancy or otherwise of these proteins. Do they compete for access to all methylated sites and therefore functionally back each other up? Or does each protein act at a subset of genes that are adapted to respond specifically to its particular regulatory influence? The answer to these questions is not yet clear, although we know that combining Mecp 2 and $M b d 2$ mutations does not lead to an obvious "synthetic" enhancement of phenotype (Guy et al. 2001). Chromatin immunopre- 
cipitation experiments identified some loci that bound to a single member of the family and other loci that appeared to bind multiple members (Ballestar et al. 2003).

\section{METHYL-CPG-BINDING PROTEINS AND DISEASE}

Dramatic demonstration of the medical relevance of methyl-CpG-binding proteins came with the discovery that at least $80 \%$ of patients with Rett Syndrome have new mutations in the MECP2 gene (Amir et al. 1999; Shahbazian and Zoghbi 2002; Kriaucionis and Bird 2003). Rett Syndrome affects females that are heterozygous for the mutations. Because of $\mathrm{X}$ chromosome inactivation, the patients are mosaic for expression of either the mutant or the wild-type gene. They develop apparently normally for 6-18 months, at which time they show regression of motor skills, repetitive hand movements, abnormal breathing, microcephaly, and other symptoms. Given the role of $\mathrm{MeCP} 2$ as a transcriptional repressor, an obvious hypothesis to explain the disease is that genes in the brain that should be silenced by MeCP2 escape repression in its absence, leading to aberrant neuronal function. As mentioned above, the discovery that MeCP2 is involved in silencing the $B d n f$ gene, which encodes a neuronal growth factor, is compatible with this theory, although much remains to be done to connect the medical condition with misexpression of this particular gene. Meanwhile, attempts to define the molecular pathology of Rett Syndrome continue with the search for additional $\mathrm{MeCP} 2$ target genes.

The relationship between DNA methylation and cancer is the subject of much research, as well as animated debate (Baylin and Bestor 2002). At the experimental level, the connection is well illustrated by the demonstration that the Min mouse model of intestinal tumorigenesis depends on the DNA methyltransferase Dnmtl (Laird et al. 1995; Eads et al. 2002). Reduced levels of this enzyme are accompanied by a significant drop in tumorigenesis, virtually abolishing the tumor-susceptible phenotype at the lowest enzyme levels compatible with viability. Parallel findings have been made in $M b d 2$ mutant animals when crossed onto a Min background (Sansom et al. 2003). Mbd2-/- Min mice develop very few tumors and live considerably longer that $M b d 2+/+$ controls. $M b d 2+/-$ heterozygotes show an intermediate phenotype, stressing the sensitivity of the assay to full dosage of Mbd2. The requirements of tumorigenesis for a protein that methylates DNA at $\mathrm{CpG}$ and also for a protein that binds to the resulting methyl-CpGs begs an important question: Which pathways to cancer involve these proteins? At present we do not know, but the disturbance of the Wnt signalling pathway in cells that have lost both copies of the Apc gene (Fearnhead et al. 2001) raises the possibility that misregulation of downstream genes is somehow involved. A significant difference between Dnmt1 and Mbd2 mutant phenotypes is that the former is an embryonic lethal whereas the latter results in viable and fertile mice. Thus both the tumor and its host depend on Dnmt1 function, but only tumors depend on Mbd2. This distinction has therapeutic implications that deserve to be pursued.

\section{CONCLUSIONS}

DNA methylation can be considered together with histone modifications as a mechanism for adapting chromatin structure to local functional needs. Like histone modifications, DNA methylation can be read by binding proteins. Methyl-CpG-binding proteins were initially characterized in model reporter gene systems, but gene knockouts in mice have begun to functionally relate each protein to specific target genes. In doing so, the mouse studies have illuminated the relationships between methyl-CpG-binding proteins and human disease.

\section{ACKNOWLEDGMENTS}

Our research is supported by the Wellcome Trust, United Kingdom.

\section{REFERENCES}

Amir R.E., Van den Veyver I.B., Wan M., Tran C.Q., Francke U., and Zoghbi H.Y. 1999. Rett syndrome is caused by mutations in X-linked MECP2, encoding methyl-CpG-binding protein 2. Nat. Genet. 23: 185.

Ballestar E., Paz M.F., Valle L., Wei S., Fraga M.F., Espada J., Cigudosa J.C., Huang T.H., and Esteller M. 2003. Methyl$\mathrm{CpG}$ binding proteins identify novel sites of epigenetic inactivation in human cancer. EMBO J. 22: 6335.

Bannister A.J., Zegerman P., Partridge J.F., Miska E.A., Thomas J.O., Allshire R.C., and Kouzarides T. 2001. Selective recognition of methylated lysine 9 on histone H3 by the HP1 chromo domain. Nature 410: 120.

Baylin S. and Bestor T.H. 2002. Altered methylation patterns in cancer cell genomes: Cause or consequence? Cancer Cell 1: 299.

Bell A.C. and Felsenfeld G. 2000. Methylation of a CTCF-dependent boundary controls imprinted expression of the Igf2 gene. Nature 405: 482.

Bird A.P. 1980. DNA methylation and the frequency of $\mathrm{CpG}$ in animal DNA. Nucleic Acids Res. 8: 1499.

Bird A. and Wolffe A.P. 1999. Methylation-induced repression-Belts, braces and chromatin. Cell 99: 451.

Boyes J. and Bird A. 1991. DNA methylation inhibits transcription indirectly via a methyl-CpG binding protein. Cell 64: 1123.

Chen R.Z., Akbarian S., Tudor M., and Jaenisch R. 2001. Deficiency of methyl-CpG binding protein-2 in CNS neurons results in a Rett-like phenotype in mice. Nat. Genet. 27: 327.

Chen W.G., Chang Q., Lin Y., Meissner A., West A.E., Griffith E.C., Jaenisch R., and Greenberg M.E. 2003. Derepression of BDNF transcription involves calcium-dependent phosphorylation of MeCP2. Science 302: 885.

Coulondre C., Miller J.H., Farabough P.J., and Gilbert W. 1978. Molecular basis of base substitution hotspots in Escherichia coli. Nature 274: 775.

Cross S.H., Meehan R.R., Nan X., and Bird A. 1997. A component of the transcriptional repressor MeCP1 is related to mammalian DNA methyltransferase and trithorax-like protein. Nat. Genet. 16: 256.

Daniel J.M. and Reynolds A.B. 1999. The catenin p120(ctn) interacts with Kaiso, a novel BTB/POZ domain zinc finger transcription factor. Mol. Cell. Biol. 19: 3614.

Daniel J.M., Spring C.M., Crawford H.C., Reynolds A.B., and Baig A. 2002. The p120(ctn)-binding partner Kaiso is a bimodal DNA-binding protein that recognizes both a sequencespecific consensus and methylated $\mathrm{CpG}$ dinucleotides. $\mathrm{Nu}$ cleic Acids Res. 30: 2911.

Eads C.A., Nickel A.E., and Laird P.W. 2002. Complete genetic suppression of polyp formation and reduction of CpG-island hypermethylation in Apc(Min/+) Dnmt1-hypomorphic mice. Cancer Res. 62: 1296. 
Fearnhead N.S., Britton M.P., and Bodmer W.F. 2001. The ABC of APC. Hum. Mol. Genet. 10: 721.

Feng Q. and Zhang Y. 2001. The MeCP1 complex represses transcription through preferential binding, remodeling, and deacetylating methylated nucleosomes. Genes Dev. 15: 827.

Fujita N., Watanabe S., Ichimura T., Ohkuma Y., Chiba T., Saya H., and Nakao M. 2003. MCAF mediates MBD1-dependent transcriptional repression. Mol. Cell. Biol. 23: 2834.

Guy J., Hendrich B., Holmes M., Martin J.E., and Bird A. 2001. A mouse Mecp2-null mutation causes neurological symptoms that mimic Rett syndrome. Nat. Genet. 27: 322.

Hark A.T., Schoenherr C.J., Katz D.J., Ingram R.S., Levorse J.M., and Tilghman S.M. 2000. CTCF mediates methylationsensitive enhancer-blocking activity at the H19/Igf2 locus. Nature 405: 486.

Hendrich B. and Bird A. 1998. Identification and characterization of a family of mammalian methyl-CpG binding proteins. Mol. Cell. Biol. 18: 6538.

Hendrich B., Guy J., Ramsahoye B., Wilson V.A., and Bird A. 2001. Closely related proteins Mbd2 and Mbd3 play distinctive but interacting roles in mouse development. Genes Dev. 15: 710 .

Hendrich B., Hardeland U., Ng H.-H., Jiricny J., and Bird A. 1999. The thymine glycosylase MBD4 can bind to the product of deamination at methylated CpG sites. Nature 401: 301.

Hutchins A., Mullen A., Lee H., Barner K., High F., Hendrich B., Bird A., and Reiner S. 2002. Gene silencing quantitatively controls the function of a developmental trans-activator. Mol. Cell 10: 81

Jenuwein T. and Allis C.D. 2001. Translating the histone code. Science 293: 1074

Jones P.L., Veenstra G.J., Wade P.A., Vermaak D., Kass S.U., Landsberger N., Strouboulis J., and Wolffe A.P. 1998. Methylated DNA and MeCP2 recruit histone deacetylase to repress transcription. Nat. Genet. 19: 187.

Jorgensen H.F., Ben-Porath I., and Bird A.P. 2004. Mbd1 is recruited to both methylated and nonmethylated CpGs via distinct DNA binding domains. Mol. Cell. Biol. 24: 3387.

Kriaucionis S. and Bird A. 2003. DNA methylation and Rett syndrome. Hum. Mol. Genet. (Spec. No. 2) 12: R221.

Lachner M., O'Carroll D., Rea S., Mechtler K., and Jenuwein T. 2001. Methylation of histone H3 lysine 9 creates a binding site for HP1 proteins. Nature 410: 116.

Laird P.W., Jackson-Grusby, Fazeli A., Dickinson S.L., Jung W.E., Li E., Weinberg R.A., and Jaenisch R. 1995. Suppression of intestinal neoplasia by DNA hypomethylation. Cell 81: 197.

Levine A., Cantoni G.L., and Razin A. 1992. Methylation in the preinitiation domain suppresses gene transcription by an indirect mechanism. Proc. Natl. Acad. Sci. 89: 10119.

Lewis J.D., Meehan R.R., Henzel W.J., Maurer-Fogy I., Jeppesen P., Klein F., and Bird A. 1992. Purification, sequence and cellular localisation of a novel chromosomal protein that binds to methylated DNA. Cell 69: 905.

Li E., Bestor T.H., and Jaenisch R. 1992. Targeted mutation of the DNA methyltransferase gene results in embryonic lethality. Cell 69: 915.

Lin X. and Nelson W.G. 2003. Methyl-CpG-binding domain protein-2 mediates transcriptional repression associated with hypermethylated GSTP1 CpG islands in MCF-7 breast cancer cells. Cancer Res. 63: 498.

Martinowich K., Hattori D., Wu H., Fouse S., He F., Hu Y., Fan G., and Sun Y.E. 2003. DNA methylation-related chromatin remodeling in activity-dependent BDNF gene regulation. Science 302: 890.

Meehan R.R., Lewis J.D., McKay S., Kleiner E.L., and Bird A.P. 1989. Identification of a mammalian protein that binds specif- ically to DNA containing methylated CpGs. Cell 58: 499.

Millar C.B., Guy J., Sansom O.J., Selfridge J., MacDougall E., Hendrich B., Keightley P.D., Bishop S.M., Clarke A.R., and Bird A. 2002. Enhanced CpG mutability and tumorigenesis in MBD4-deficient mice. Science 297: 403.

Nan X., Campoy J., and Bird A. 1997. MeCP2 is a transcriptional repressor with abundant binding sites in genomic chromatin. Cell 88: 471.

Nan X., Meehan R.R., and Bird A. 1993. Dissection of the methyl-CpG binding domain from the chromosomal protein MeCP2. Nucleic Acids Res. 21: 4886.

Nan X., Tate P., Li E., and Bird A.P. 1996. DNA methylation specifies chromosomal localization of MeCP2. Mol. Cell. Biol. 16: 414 .

Nan X., Ng H.-H., Johnson C.A., Laherty C.D., Turner B.M., Eisenman R.N., and Bird A. 1998. Transcriptional repression by the methyl-CpG-binding protein MeCP2 involves a histone deacetylase complex. Nature 393: 386.

Ng H.-H., Zhang Y., Hendrich B., Johnson C.A., Burner B.M., Erdjument-Bromage H., Tempst P., Reinberg D., and Bird A. 1999. MBD2 is a transcriptional repressor belonging to the MeCP1 histone deacetylase complex. Nat. Genet. 23: 58.

Ohki I., Shimotake N., Fujita N., Nakao M., and Shirakawa M. 1999. Solution structure of the methyl-CpG-binding domain of the methylation-dependent transcriptional repressor MBD1. EMBO J. 18: 6653.

Ohki I., Shimotake N., Fujita N., Jee J., Ikegami T., Nakao M., and Shirakawa M. 2001. Solution structure of the methyl$\mathrm{CpG}$ binding domain of human MBD1 in complex with methylated DNA. Cell 105: 487.

Prokhortchouk A., Hendrich B., Jorgensen H., Ruzov A., Wilm M., Georgiev G., Bird A., and Prokhortchouk E. 2001. The p120 catenin partner Kaiso is a DNA methylation-dependent transcriptional repressor. Genes Dev. 15: 1613.

Sansom O.J., Berger J., Bishop S.M., Hendrich B., Bird A., and Clarke A.R. 2003. Deficiency of Mbd2 suppresses intestinal tumorigenesis. Nat. Genet. 34: 145.

Shahbazian M.D. and Zoghbi H.Y. 2002. Rett syndrome and $\mathrm{MeCP} 2$ : Linking epigenetics and neuronal function. Am. J. Hum. Genet. 71: 1259 .

Shahbazian M.D., Antalffy B., Armstrong D.L., and Zoghbi H.Y. 2002. Insight into Rett syndrome: MeCP2 levels display tissue- and cell-specific differences and correlate with neuronal maturation. Hum. Mol. Genet. 11: 115.

Stancheva I., Collins A.L., Van den Veyver I.B., Zoghbi H., and Meehan R.R. 2003. A mutant form of MeCP2 protein associated with human Rett syndrome cannot be displaced from methylated DNA by notch in Xenopus embryos. Mol. Cell 12: 425.

Tudor M., Akbarian S., Chen R.Z., and Jaenisch R. 2002. Transcriptional profiling of a mouse model for Rett syndrome reveals subtle transcriptional changes in the brain. Proc. Natl. Acad. Sci. 99: 15536.

Wade P.A., Gegonne A., Jones P.L., Ballestar E., Aubry F., and Wolffe A.P. 1999. Mi-2 complex couples DNA methylation to chromatin remodelling and histone deacetylation. Nat. Genet. 23: 62.

Wakefield R.I.D., Smith B.P., Nan X., Free A., Soterious A., Uhrin D., Bird A., and Barlow P.N. 1999. The solution structure of the domain from MeCP2 that binds to methylated DNA. J. Mol. Biol. 291: 1055.

Winston F. and Allis C.D. 1999. The bromodomain: A chromatin-targeting module? Nat. Struct. Biol. 6: 601.

Yoon H.G., Chan D.W., Reynolds A.B., Qin J., and Wong J. 2003. N-CoR mediates DNA methylation-dependent repression through a methyl $\mathrm{CpG}$ binding protein Kaiso. Mol. Cell 12: 723 . 


\title{
$8_{\mathrm{CSH}}^{\infty} \mathrm{H} \&$ Cold Spring Harbor Symposia SYMPOSIA
}

\section{Reading the DNA Methylation Signal}

\author{
A. BIRD and D. MACLEOD
}

Cold Spring Harb Symp Quant Biol 2004 69: 113-118

Access the most recent version at doi:10.1101/sqb.2004.69.113

References This article cites 54 articles, 18 of which can be accessed free at: http://symposium.cshlp.org/content/69/113.full.html\#ref-list-1

\section{License}

Email Alerting Receive free email alerts when new articles cite this article - sign up in Service the box at the top right corner of the article or click here. 